\title{
Determinación precisa de posición y orientación relativa en satélites de arquitectura segmentada
}

\section{Precise determination of relative position and orientation in segmented architecture satellites}

Presentación: 22/11/2019

\section{Doctorando:}

\section{Ing. Marco Alvarez Reyna}

Universidad Tecnológica Nacional, Facultad Regional Córdoba

malvarez@frc.utn.edu.ar

\section{Director/es:}

\section{Dr. Julián Pucheta \\ Dr. Juan Fraire}

\section{Resumen}

El tema general de esta Tesis Doctoral es navegación, guiado y control para Sistemas Aeroespaciales Distribuidos (SAD). Los Satélites de Arquitectura Segmentada (SAS) son un ejemplo de SAD, donde dos o más segmentos (satélites pequeños y simples) cumplen una función similar a la de un satélite monolítico tradicional. Algunos términos relacionados con SAS son: vuelo en formación [3], redes espaciales, instrumentos partidos y navegación en clúster, siendo este último el tema particular de la presente investigación. Ésta propone centrarse en aspectos relacionados con la navegación relativa (determinación precisa de la órbita y orientación relativas a bordo) entre los segmentos, y su uso para guiarlos y controlarlos de forma autónoma.

Palabras claves: navegación en clúster, órbita, orientación, procesamiento a bordo, guiado y control automático

\begin{abstract}
The general topic of this $\mathrm{PhD}$ Thesis is about navigation, guidance and control for Distributed Aerospace Systems (DAS). Segmented Architecture Satellites (SAS) are an example of DAS, were a couple or more segments (small and simple satellites) do a similar work than a big and traditional monolithic satellite. Terms related to SAS are: formation flying, space networks, parted instruments and cluster navigation, the latter being the particular topic of this Doctoral research. It proposes to focus on aspects related to the relative navigation (onboard precise determination of relative orbit and orientation) between the segments and their use in guiding and controlling them autonomously.
\end{abstract}

Keywords: cluster navigation, orbit, attitude, onboard proccessing, autonomous guidanec and control

\section{Introducción}

La presente investigación Doctoral aborda las Misiones Espaciales Satelitales a partir de un nuevo paradigma [1]: Satélites de Arquitectura Segmentada (SAS) como parte de un Sistema Aeroespacial Distribuido (SAD).

Una misión espacial-satelital de arquitectura segmentada propone distribuir las funciones tanto de la plataforma de servicio como del módulo de carga útil [1][4] en distintos satélites que se agrupan formando un clúster. 
Cada uno de los satélites que conforman el clúster es llamado segmento. Cabe aclarar que si bien en general se hace referencia a los segmentos como segmentos de vuelo, en el paradigma de arquitectura segmentada una estación terrena también puede ser considerada como un segmento mas del sistema. Así mismo, un segmento puede estar constituido por dos o mas nodos.

El vuelo en clúster conlleva un gran esfuerzo de coordinación en la operación de los segmentos, por lo que la tendencia es a automatizar procesos vinculados a la prevención de colisiones, acoplamiento, reposicionamiento dentro de la constelación y posicionamiento preciso del instrumento distribuido.

Esto último implica un desafío tecnológico muy importante: controlar la posición relativa de los segmentos con extrema precisión.

Una de las principales tecnologías habilitantes asociada a este desafío tecnológico es la navegación en clúster.

La presente investigación Doctoral propone centrarse en aspectos referentes a la navegación relativa entre los segmentos y su utilización en el guiado y control de los mismos.

Los sistemas de guiado y control son consumidores de los datos generados por el sistema de navegación, por lo que modernamente se abordan las tres áreas como una sola temática general, permitiendo adquirir una visión sistémica del problema. De esta manera, introducirse en las disciplinas de guiado y control resulta fundamental para el desarrollo de la presente propuesta de Tesis Doctoral.

La navegación relativa típicamente se sirve de filtros para combinar diferentes fuentes de información y tipos de datos provenientes de sensores con niveles de precisión muy diferentes, para estimar los vectores de estado de los vehículos en tiempo real, por lo que se implementará la fusión de varios sensores a fin de obtener algoritmos de navegación con performance tal que contribuyan a avanzar el estado del arte en la presente temática.

Para misiones de vuelo en clúster, ya sean cooperativas o no cooperativas, los requerimientos de precisión en los sistemas de navegación son usualmente más altos que para misiones donde un sólo satélite está involucrado. En potenciales aplicaciones como interferometría, o gravimetría, se puede requerir altísima precisión [3] en posición y velocidad relativa. Por ejemplo, si se considera docking entre dos vehículos, los requerimientos pueden definirse en términos centímetros o milímetros de error en posición relativa.

Se espera que la tecnología de navegación en clúster permita ofrecer mejores prestaciones en la determinación precisa del estado del los segmentos y habilitar tanto el desarrollo de instrumentos partidos como también de sistemas aeroespaciales distribuidos. En particular los instrumentos partidos permitirían obtener información de origen espacial que de otra manera sería muy complicada y costosa de lograr con las tecnologías actuales.

\section{Resultados}

\section{Derivados del estudio del estado del arte:}

Como fruto del estudio del estado del arte se llega a una primer conclusión, preliminar, sobre la problemática a resolver: ¿Cómo resolver la navegación relativa partiendo de un clúster con 2 segmentos generalizando la solución para un clúster con tres segmentos? Planteándose un escenario de prueba donde el conjunto mínimo de segmentos del clúster sea $\mathrm{N}=3^{*} \mathrm{n}(\mathrm{c} / \mathrm{n}: 1,2,3)$.

Así mismo se proponen dos instrumentos partidos como aplicaciones candidatas a condicionar los requerimientos de un SAD compuesto por SAS, y son: Hiper resolución (en espectro visible, infrarrojo; hiper espectral); Antena partida (SAR, geo localización, comunicaciones); Interferometría y gravimetría.

Otras de las conclusiones devengadas del estudio del estado del arte es que, dado que se seleccione como fuente de datos secundaria para fusión a un sistema de adquisición de imágenes a bordo, hay una solución de compromiso [6] a la hora de elegir entre visión estéreo [7], que entrega mas robustez y precisión, contra visión mono, que tiene las ventajas de consumir menos energía, espacio y volumen, y menor probabilidad de falla (menos elementos constituyentes del sistema). Una alternativa a explorar ería la posibilidad de generar visión estéreo a partir de un único sensor matricial y un diseño óptico que permita generar las imágenes Iz+De. 
Finalmente se plantea el siguiente interrogante: ¿En qué medida la dinámica de las comunicaciones entre los segmentos del clúster pasa formar parte del modelo de determinación de órbita y orientación relativas?

\section{Derivados de las primeras instancias de exploración e investigación:}

Dado que los SAD están comenzando a desarrollarse, no hay un estándar de definiciones en torno a las distintas técnicas, configuraciones, y nombres en general, por lo que se trabajó en un conjunto de definiciones para poder hacer referencia en este trabajo. Así mismo se trabajó en la exploración de las distintas tecnologías habilitantes de este nuevo paradigma de los SAD.

Se avanzó en la definición en alto nivel de requerimientos de un SAS, principalmente observando la característica de navegación autónoma.

Se inició el estudio sobre modelado de sensores y actuadores, particularmente Gyros, StarTracker (a partir de Gyros), y GPS [2] (a partir de órbita). Complementariamente se está explorando la posibilidad de incorporar HIL y SIL al esquema de sensores y actuadores, hay avances relativos sobre HIL para Gyro (podría ser utilizado para cerrar un lazo de determinación de orientación fusionando con visión artificial desde otro segmento).

Se avanzó hasta la experimentación con el simulador de dinámica traslacional (órbita), teniendo en cuenta perturbaciones gravitacionales hasta J2 [5]. Como producto derivado se trabajó en un algoritmo de transformación de sistema de coordenadas geográfica LLH (GPS) a XYZ y en un método de validación de propagadores orbitales y TLE, basado en telemetría adquirida por GPS en vuelo tema candidato a una publicación). Los resultados obtenidos muestran que es posible comparar las estimaciones con datos medidos y se observa un patrón de error que evoluciona en el tiempo como era esperado, debido a la divergencia natural del modelo respecto de los datos medidos, en función del tiempo.

Se inició el estudio sobre simulación de dinámica rotacional (orientación).

Se inició el estudio sobre los modelos de movimiento relativo, basado escenarios.

Sobre el filtro de navegación distribuido: se iniciaron estudios sobre comunicaciones satelitales, iniciando por los flujos de análisis y diseño de un radio enlace tierra-satélite (up/down link). Diagramas y modelos computacionales desarrollados y en etapa de revisión.

\section{Referencias}

[1] Brown, O., \& Eremenko, P. (2006). Fractionated space architectures: a vision for responsive space. DEFENSE ADVANCED RESEARCH PROJECTS AGENCY ARLINGTON VA.

[2] http://www.gps.gov/

[3] Alfriend, K., Vadali, S. R., Gurfil, P., How, J., \& Breger, L. (2009). Spacecraft formation flying: Dynamics, control and navigation (Vol. 2). Butterworth-Heinemann.

[4] Ley, W., Wittmann, K., \& Hallmann, W. (Eds.). (2009). Handbook of space technology (Vol. 22). John Wiley \& Sons.

[5] Curtis, H. (2013). Orbital mechanics for engineering students. Butterworth-Heinemann.

[6] Segal, S., Gurfil, P., \& Shahid, K. (2014). In-orbit tracking of resident space objects: A comparison of monocular and stereoscopic vision. IEEE Transactions on Aerospace and Electronic Systems, 50(1), 676-688.

[7] Segal, S., Carmi, A., \& Gurfil, P. (2014). Stereovision-based estimation of relative dynamics between noncooperative satellites: theory and experiments. IEEE Transactions on Control Systems Technology, 22(2), 568-584. 